\title{
A spin-statistics theorem for certain topological geons
}

\author{
H F Dowker†§ and R D Sorkin $\ddagger$ \\ $\dagger$ Lauritsen Laboratory, California Institute of Technology, Pasadena, CA 91125, USA \\ $\ddagger$ Instituto de Ciencias Nucleares, UNAM, A Postal 70-543, DF 04510, Mexico \\ and \\ Physics Department, Syracuse University, Syracuse, NY 13244-1130, USA
}

Received 8 December 1997

\begin{abstract}
We review the mechanism in quantum gravity whereby topological geons, particles made from non-trivial spatial topology, are endowed with non-trivial spin and statistics. In a theory without topology change there is no obstruction to 'anomalous' spin-statistics pairings for geons. However, in a sum-over-histories formulation including topology change, we show that non-chiral Abelian geons do satisfy a spin-statistics correlation if they are described by a wavefunction which is given by a functional integral over metrics on a particular 4-manifold. This manifold describes a topology changing process which creates a pair of geons from $\mathbb{R}^{3}$.
\end{abstract}

PACS numbers: 0460, 0460G, 0240, 0530

\section{Introduction}

When the configuration space of a classical system is non-simply connected (or, more generally, non-contractible) it allows for a richer variety of possibilities quantum mechanically than usual. In particular, the possibility arises for 'emergent' fermionic statistics and spinorial (half-odd-integral) spin for objects built from fields which are fundamentally tensorial (integral spin) and bosonic. General relativity is such a classical theory and in quantum general relativity on a product spacetime manifold $\mathbb{R} \times{ }^{3} M$ it can be shown that topological geons may be endowed with both non-trivial spin [1] and non-trivial statistics [2].

In nature, the spin and statistics of all known particles are correlated: they are bosons if and only if they are tensorial, and fermions if and only if they are spinorial. Quantum geons, on the other hand, satisfy no such correlation in the canonical theory: any combination of spin and statistics is possible [2-4]. It is perhaps not surprising that geons in canonical quantum gravity appear to violate the usual correlation. In the proofs of all existing spinstatistics theorems, the (explicit or implicit) possibility of particle-antiparticle creation (and annihilation) is crucial. But the process of geon-antigeon pair production is a topology changing one and cannot be described within a formalism which assumes a priori that the spatial 3-manifold is fixed. It has therefore been conjectured that in a formulation of quantum gravity which can accommodate topology change, the usual spin-statistics connection would be recovered for geons [3].

One such formulation is the sum over histories $(\mathrm{SOH})$ with inclusion of non-product spacetime manifolds. In this paper we will show that for certain geons, a spin-statistics

$\S$ Current address: Blackett Laboratory, Imperial College, London SW7 2BZ, UK.

0264-9381/98/051153+15\$19.50 (C) 1998 IOP Publishing Ltd 
theorem can be proved in this context, one which relies on the wavefunction describing the quantum state of the geons being given by a functional integral on a certain ' $U$-tube' manifold.

In section 2 we will briefly describe the way in which topological geons acquire spin and statistics. In section 3 we adopt a $\mathrm{SOH}$ approach to quantum gravity and introduce the key assumption that the geons we consider are described by a functional integral over metrics on a certain 4-manifold, ${ }^{4} M$, that mediates the pair production of the geons. We will see how this implies that a certain diffeomorphism intimately connected with spin and statistics, acts trivially on the wavefunction. In section 4 we show that for a particular sort of geon, the lens spaces, this result leads to a spin statistics theorem: the lens space geons (which are necessarily tensorial) must always be bosons. More generally, the theorem we prove applies to any geon which carries an Abelian representation of its internal diffeomorphism group. Section 5 gives a summary and discussion of possible extensions of this work.

In order to avoid repeatedly having to make certain caveats, we will restrict ourselves to orientable 3-manifolds in this paper; this restriction could straightforwardly be dropped and we will mention in the final section how our results generalize to the non-orientable case. We will further assume that no handles, $S^{2} \times S^{1}$, occur in the 'prime decomposition' of the 3-manifold (see section 2).

\section{Topological geons}

In this section we briefly review the background to our problem, referring the reader to [2-4] for more details. Roughly, topological geons are particles made from non-trivial spatial topology. We will be interested in the situation of an isolated system of particles and thus we will be dealing with a three-dimensional manifold $M$ which admits asymptotically flat metrics. Physically, $M$ is 3-space at a 'moment of time', or if you prefer, the 'future boundary of truncated spacetime' [5].

\subsection{The topology}

There is a '3-manifold decomposition theorem' that identifies candidates for elementary geons, but in order to state this theorem we must first introduce the concepts of "connected sum' and 'prime manifold'. To take the connected sum of two oriented 3-manifolds $M_{1}$ and $M_{2}$, remove an open ball from each and identify the resulting 2-sphere boundaries with an orientation-reversing diffeomorphism (henceforth, 'diffeo'). Taking the connected sum of any 3-manifold with $S^{3}$ gives a manifold diffeomorphic to the original one; taking it with $\mathbb{R}^{3}$ is topologically equivalent to deleting a point. A prime 3-manifold, $P$, is a closed 3manifold that is not $S^{3}$ and such that whenever $P=P_{1} \# P_{2}$, either $P_{1}$ or $P_{2}$ is $S^{3}$. Examples of primes are the 3-torus, $T^{3}$, and the so-called spherical spaces, $S^{3} / G$, where $G$ is some discrete subgroup of $S O(4)$ acting freely on $S^{3}$.

The $M$ we are considering is $M=\mathbb{R}^{3} \# K$ where $K$ is a closed 3-manifold. The decomposition theorem states that any such $M$ can be decomposed into the connected sum of finitely many prime manifolds and this decomposition is unique:

$$
M=\mathbb{R}^{3} \# P_{1} \# P_{2} \ldots \# P_{n} .
$$

We will assume that to each prime summand there corresponds an elementary quantum geon; with 'correspond' being used in a suitable sense since there is a rather subtle relation between a particular piece of spatial topology and a physical particle, the subtlety being 
related to both familiar 'identical particle exchange effects' and unfamiliar effects due to the existence of diffeos known as 'slides' [3, 4].

A very useful way to visualize a multi-geon manifold relies on the result that any prime manifold (in fact, any closed 3-manifold) can be constructed from a solid convex polyhedron by performing appropriate identifications on its faces. For example, the 3-torus prime, $T^{3}$, is made by identifying opposite faces of a solid cube. The spherical space $S^{3} / Q$, where $Q$ is the eight-element quaternion subgroup of $S U(2)$, is also made from a solid cube, this time identifying opposite faces after a $\frac{1}{2} \pi$ rotation. Suppose $M=\mathbb{R}^{3} \# P$ where $P$ is a prime expressed as a certain solid polyhedron with identifications. Then $M$ is diffeomorphic to $P \backslash\{$ point $\}$. By letting the point be removed from the interior of $P$ and imagining 'turning $P$ inside out', one sees that $M$ can be constructed by deleting the same (open) solid polyhedron from $\mathbb{R}^{3}$ and making the same identifications on the boundary. In the same way the multi-geon manifold $M=\mathbb{R}^{3} \# P_{1} \# P_{2} \# \ldots \# P_{n}$ can be made by cutting out an appropriate polyhedron from $\mathbb{R}^{3}$ for each summand and making appropriate identifications.

\subsection{Wavefunctions and the mapping class group}

In canonical quantum gravity, for which the topology does not change, the configuration space, $Q$, is the space of all 3-geometries on $M$,

$$
Q=\frac{\operatorname{Riem}^{\infty}(M)}{\operatorname{Diff}^{\infty}(M)}
$$

where $\operatorname{Riem}^{\infty}(M)\left(\mathrm{R}^{\infty}\right.$ for short) is the space of asymptotically flat Riemannian metrics on $M$ and $\operatorname{Diff}^{\infty}(M)$ ( $\mathrm{D}^{\infty}$ for short) is the group of diffeomorphisms of $M$ that become trivial on approach to infinity†. It can be shown that $\mathrm{D}^{\infty}$ acts freely on $\mathrm{R}^{\infty}$ and so $Q$ is a manifold, $\mathrm{R}^{\infty}$ being a principal fibre bundle over $Q$ with fibre $\mathrm{D}^{\infty}$. Thus, using the fact that $\mathrm{R}^{\infty}$ is convex and hence contractible to a point so that all its homotopy groups are trivial, we deduce that $\pi_{k}\left(\mathrm{D}^{\infty}\right) \simeq \pi_{k+1}(Q)$.

Wavefunctions need not be single-valued on $Q$ if $Q$ contains non-contractible loops. Rather, the transformation of a wavefunction as such loops are traversed gives a representation of $\pi_{1}(Q)$. This is a special case of the general situation where wavefunctions are sections of a twisted vector bundle on $Q$.

In the so-called covering space quantization, wavefunctions can be represented as (single-valued) complex functions on the universal covering space of $Q$,

$$
\tilde{Q}=\frac{\operatorname{Riem}^{\infty}(M)}{\operatorname{Diff}_{0}^{\infty}(M)}
$$

where $\operatorname{Diff}_{0}^{\infty}(M) \subset \operatorname{Diff}^{\infty}(M)$ is the connected component of the identity. The space $\tilde{Q}$ is a principal fibre bundle over $Q$ with fibre $G=\pi_{0}\left(\mathrm{D}^{\infty}\right):=\mathrm{D}^{\infty} / \mathrm{D}_{0}^{\infty}$

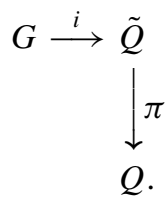

The group $G$, known as the mapping class group (MCG) of $M$, acts (globally) on the right on $\tilde{Q}$ : if $g \in G$ and $\tilde{q} \in \tilde{Q}$ then, under the action of $g, \tilde{q} \mapsto \tilde{q} g$. This action can be

$\dagger$ By asymptotically flat we mean asymptotic to some fixed, flat metric in a neighbourhood of infinity. The exact space $Q$ will depend on the precise falloff conditions imposed on the metrics and diffeomorphisms, but we will only be interested here in topological properties of all the spaces which are insensitive to the choice of conditions. 
given in terms of metrics and diffeomorphisms as follows. Let $h$ be some representative metric of the equivalence class $\tilde{q}, \tilde{q}=[h]$, and $d$ some representative diffeomorphism of the equivalence class $g$. Then

$$
\tilde{q} g=\left[d^{*}(h)\right]
$$

where $d^{*}(h)$ is the pullback of $h$ by $d$. The action induces an action on functions on $\tilde{Q}$ by the requirement that they transform as scalars. More specifically, under $g, \Psi \mapsto \Psi g$ where

$$
(\Psi g)(\tilde{q})=\Psi\left(\tilde{q} g^{-1}\right) .
$$

Notice our convention of writing the action of $g$ on the right of $\Psi$ to agree with its action on $\tilde{Q}$.

If, now, we consider square-summable functions $\psi$ on a single fibre of the covering space $\tilde{Q}$, then (2.6) says precisely that the space of such functions carries the regular representation $R$ of $G$. When $G$ is a finite group, every unitary irreducible representation of $G$ occurs as a subrepresentation of $R$, and conversely $R$ can be decomposed uniquely as a sum of irreducibles. Physically each such irreducible corresponds to a distinct sector ('theta sector') of the quantum theory, and these sectors will be superselected if topology change is ignored. (Note that, since an irreducible of dimension $d$ occurs $d$ times in $R$, there will be more irreducible subspaces than physically distinct sectors. See [2] on this point.) When $G$ is infinite (which the MCG almost always is), these statements about irreducibles must be replaced by a considerably more complicated set of assertions [6]. In part, this is just the familiar problem of delta-function normalization for eigenvectors of operators with a continuous spectrum, but that is not the whole story. Nevertheless, we believe that it remains formally true that every irreducible can, in an appropriate sense, be obtained from the regular representation, at least if we limit ourselves to irreducibles of finite dimension. We return briefly to this point near the end of the paper, where we argue that the difficulty belongs to the wavefunction rather than the physics, and will disappear if one adheres consistently to a sum-over-histories formulation.

Equation (2.6) defines an action of $G$ on the space of wavefunctions on $\tilde{Q}$. For consideration of spin and statistics properties we are actually interested in the action of loops in $Q$ (elements of $\pi_{1}(Q)$ ) on wavefunctions. Although $G$ is isomorphic to $\pi_{1}(Q)$ the isomorphism is not canonical. Another way to say this is that diffeos act (globally) on the right on the bundle $\tilde{Q}$, whereas loops act (locally) on the left. Let us see what this means in more detail by constructing an isomorphism from $\pi_{1}(Q)$ to $G$.

More specifically, let $\pi_{1}(Q)=\pi_{1}\left(Q ; q_{0}\right)$ be the first homotopy group based at $q_{0} \in Q$, and let $\gamma$ be a representative of the homotopy class $[\gamma] \in \pi_{1}\left(Q ; q_{0}\right)$. Now $[\gamma]$ induces an automorphism of $\pi^{-1}\left(q_{0}\right)$, the fibre above $q_{0}$, by sending the point $\tilde{q} \in \pi^{-1}\left(q_{0}\right)$ to the point at the end of the path in $\tilde{Q}$ which is the unique lift of $\gamma$ that begins at $\tilde{q}$. Choose a fiducial element, $\tilde{q}_{0} \in \pi^{-1}\left(q_{0}\right)$, then $\pi^{-1}\left(q_{0}\right)=\left\{\tilde{q}_{0} f: f \in G\right\}$, and the requirement

$$
[\gamma]: \tilde{q}_{0} \mapsto \tilde{q}_{0} g_{\gamma}
$$

sets up a correspondence $\Phi$ between $[\gamma] \in \pi_{1}(Q)$ and $g_{\gamma} \in G$ which depends on the choice of $\tilde{q}_{0}$ (but not on the choice of $\gamma$ to represent $[\gamma]$ ). Now the action of the loop $\gamma$ on a general point on the fibre, $\tilde{q}=\tilde{q}_{0} f_{\tilde{q}}$ with $f_{\tilde{q}} \in G$, is

$$
[\gamma]: \tilde{q}=\tilde{q}_{0} f_{\tilde{q}} \mapsto[\gamma]\left(\tilde{q}_{0} f_{\tilde{q}}\right)=\left([\gamma] \tilde{q}_{0}\right) f_{\tilde{q}}=\tilde{q}_{0} g_{\gamma} f_{\tilde{q}}\left[=\tilde{q}\left(f_{\tilde{q}}^{-1} g_{\gamma} f_{\tilde{q}}\right)\right]
$$

from which we can deduce that (i) $\Phi:[\gamma] \mapsto g_{\gamma}$ is an isomorphism and (ii) the isomorphism is not canonical since choosing a different fiducial point on the fibre gives a different isomorphism (related by conjugation). 
Let $\Psi$ be a wavefunction on $\tilde{Q}$ with support on the fibre $\pi^{-1}\left(q_{0}\right)$. The action via (2.8) of $\pi_{1}(Q)$ on this fibre induces an action on $\Psi, \Psi \mapsto[\gamma] \Psi$, where

$$
[\gamma] \Psi(\tilde{q})=\Psi\left(\tilde{q}_{0} g_{\gamma}^{-1} f_{\tilde{q}}\right)
$$

(Compare this to (2.6).) We can extend all of this to a neighbourhood of the fibre in which the bundle is a product, since a loop through $q_{0}$ maps unambiguously to a loop at a neighbouring point $q_{0}^{\prime}$ within such a neighbourhood. Notice that the loop $[\gamma]$ acts trivially on a wavefunction $\Psi$ (i.e. leaves it invariant) if the diffeo $g_{\gamma}$ and all conjugates of it, $g g_{\gamma} g^{-1}$, act trivially on $\Psi$.

As we have already done, we will often refer, imprecisely, to an element of $\pi_{0}\left(\mathrm{D}^{\infty}\right)$ as a diffeo and an element of $\pi_{1}(Q)$ as a loop but there should be no ambiguity involved. We will actually never be interested in loops and diffeos as such but only in their homotopy and isotopy classes.

\subsection{Spin and statistics}

Now let $M=\mathbb{R}^{3} \# P \# P$ and let $q \in Q$ be a configuration in which two isometric geons are sitting at well separated positions with plenty of flat space between them. Call the loop based at $q$ which describes the two geons moving (by translation) around each other until they have swapped places the 'exchange loop', $\gamma_{e}$, and the loop that describes one geon spinning around by $2 \pi$ the ' $2 \pi$ rotation loop' of that geon, $\gamma_{2 \pi}^{i}$, where $i=1,2$ labels the geon (by its physical position). Further, suppose we have a wavefunction, $\Psi$, on $\tilde{Q}$ which is peaked on the fibre over $q$.

If the $2 \pi$ rotation loop of one geon is represented on $\Psi$ by $1(-1)$ then that geon is tensorial (spinorial). If the exchange is represented by $1(-1)$ and the two geons are in identical internal states then the geons are bosons (fermions). In a version of quantum gravity in which the 3-manifold $M$ is fixed, there is no correlation between the spin type and statistics that geons can have. In the case of two identical primes, there exist finite-dimensional unitary irreducible representations of the MCG for each of the possible combinations: fermion-tensorial, fermion-spinorial, boson-tensorial and boson-spinorial [4].

This lack of a correlation can be attributed to the fact that in a frozen topology theory (such as the canonical quantization) there is no allowance for geon-antigeon production since a process in which a geon and antigeon are created from $\mathbb{R}^{3}$ is a topology changing one (we know this from the decomposition theorem: one piece of non-trivial topology cannot 'cancel' another). The known spin-statistics theorems for objects such as skyrmions and other kinks which have these 'emergent' properties of spin and statistics all require, for their proofs, that the process of pair creation and annihilation be describable as a path in the configuration space. For two $S U$ (2) skyrmions, for example, the exchange loop and the $2 \pi$ rotation loops in the 2 -skyrmion sector of the configuration space can be shown to be homotopic, and therefore must be represented on the state vector identically. The homotopy sequence of loops leading from the exchange to one of the $2 \pi$ rotations contains a loop which describes a skyrmion-anti-skyrmion pair emerging from the vacuum and the anti-skyrmion annihilating with one of the original skyrmions to leave two skyrmions again. See also [7] for a more general theorem. 
All this leads one to expect that in a formulation of quantum gravity in which topology change is naturally accommodated there is hope that the spin statistics correlation can be recovered $\dagger$. We will therefore turn now to the sum-over-histories $(\mathrm{SOH})$ approach.

\section{Sum over histories}

\subsection{The wavefunction}

Henceforth we take $M=\mathbb{R}^{3} \# P \# P$ where $P$ is a non-chiral prime, that is, a prime which admits an orientation reversing diffeo. Construct a 4-manifold ${ }^{4} M$ with 'initial' boundary $M_{0}=\mathbb{R}^{3}$ and 'final' boundary $M=\mathbb{R}^{3} \# P \# P$ by taking $\mathbb{R}^{3} \times I$, where $I$ is the unit interval and deleting a 'U-tube' of polyhedral cross section. Figure 1 is a depiction of this in $2+1$ dimensions - the generalization to $3+1$ should be clear.

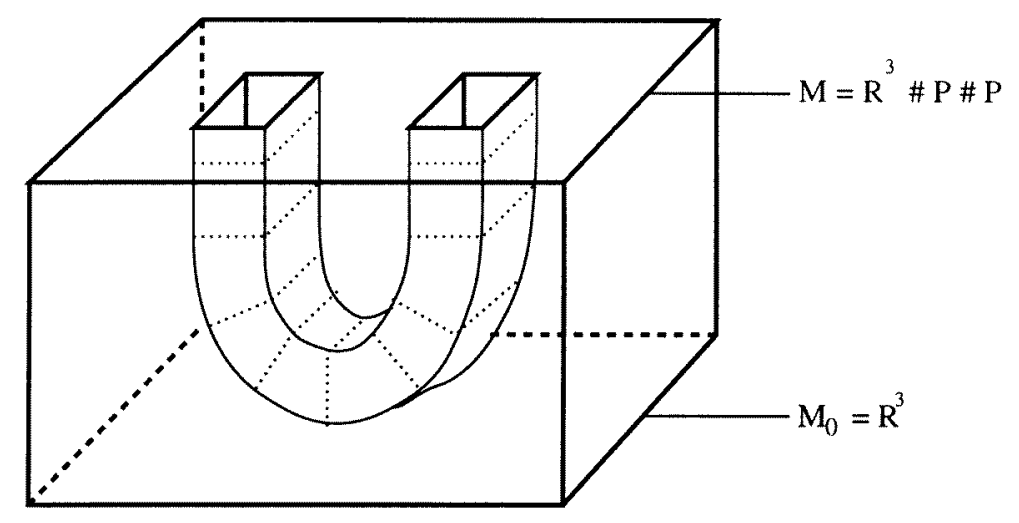

Figure 1. ${ }^{4} M$.

The tube is drawn with square (imagine cubic) cross section, appropriate for torus geons, for example; in general, the cross section will be a more complicated polyhedron. Identifications are made on each cross section (shown with dotted lines) of the cut-out tube's boundary, just as in constructing $\mathbb{R}^{3} \# P$. The condition that $P$ be non-chiral is necessary for the existence of this 4-manifold: if one end of the tube had identifications made on it that made it a chiral prime, $\hat{P}$, then the other end would be a different prime, $\hat{P}^{*}$, its 'CP conjugate'.

Consider wavefunctions given by a functional integral of the following form:

$$
\Psi(h)=\int_{\mathcal{B}}\left[\mathrm{d} h_{0}\right] \Psi_{0}\left(h_{0}\right) \int_{\mathcal{C}}[\mathrm{d} g] \mathrm{e}^{\mathrm{i} S[g]}
$$

where $\mathcal{B}$ is the class of all asymptotically flat 3-metrics on $M_{0}, \Psi_{0}$ is any wavefunction on $\mathcal{B}, \mathcal{C}$ is the class of all 4-metrics on the 4-manifold ${ }^{4} M$ which induce $h_{0}$ on $M_{0}$ and $h$ on $M$ and approach some fixed flat metric at infinity. The class $\mathcal{C}$ could be more restricted: one might want to sum over metrics with a fixed 4-volume, for example [5]. Note that $\Psi$ is given as a function on $\mathrm{R}^{\infty}$, whereas we want it to be a function on $\tilde{Q}$. In fact, it defines a function on $\tilde{Q}$ since it is constant on equivalence classes of metrics related by diffeos $j$ connected to the identity.

$\dagger$ One could take the position that the unreasonably large number of inequivalent quantum sectors which arise in the canonical theory, due to the effect of the slides discussed in subsection 3.3, is another reason to abandon the assumption of frozen topology [4]. 
This follows from the more general result that any element, $f \in \mathrm{D}^{\infty}$, that admits an extension ${ }^{4} f:{ }^{4} M \rightarrow{ }^{4} M$ which is the identity on $M_{0}$, and which tends to the identity at infinity, acts trivially on (3.1). (From now on, we will take for granted that any diffeo of ${ }^{4} M$ which we consider must tend to the identity at infinity. Also notice that since $\pi_{0}\left(\mathrm{D}^{\infty}\left(\mathbb{R}^{3}\right)\right)$ is trivial the question of whether there exists an extension which fixes $M_{0}$ pointwise reduces to the question of whether there exists any extension at all.) This holds if the 'measure factor' $[\mathrm{d} g]$ and amplitude $\mathrm{e}^{\mathrm{i} S[g]}$ in (3.1) are diffeomorphism invariant. Indeed, consider $\Psi(h)$ and $(\Psi f)(h)=\Psi\left(f^{*}(h)\right)$. If ${ }^{4} f$ exists such that its restriction to the initial boundary is the identity and to the final boundary is $f$, then for each metric $g$ contributing to $\Psi$ there is a diffeomorphic partner, ${ }^{4} f^{*}(g)$ contributing the same amount to $\Psi f$ and vice versa. Thus the two wavefunctions are equal.

Now, any diffeo, $j \in \mathrm{D}^{\infty}$, connected to the identity is extendible to a diffeo of ${ }^{4} M$ [8]. Briefly, this can be seen by constructing an extension which is the identity outside a neighbourhood of the boundary $M$; on the neighbourhood, which is diffeomorphic to $M \times[0,1]$, it is defined using the isotopy sequence of diffeos between $j$ and the identity. The wavefunction constructed in (3.1) is therefore invariant under $j$.

We should also confirm that extendibility is an isotopy invariant property, i.e. if $g, g^{\prime} \in[g]$ and $g$ is extendible to ${ }^{4} M$, then so is $g^{\prime}$. Let the extension of $g$ be ${ }^{4} g:{ }^{4} M \rightarrow{ }^{4} M$ and let the isotopy sequence be $i:[0,1] \rightarrow \mathrm{D}^{\infty}(M)$ with $i(0)=g^{\prime}$ and $i(1)=g$. Consider a closed neighbourhood, $N$, of $M$ in ${ }^{4} M$. There exist diffeos: $\chi: N \rightarrow[0,1] \times M$ and $\theta:{ }^{4} M \backslash \dot{N} \rightarrow{ }^{4} M$ which agree on $(N \backslash \dot{N})$. We define ${ }^{4} g^{\prime}$ as follows. For $x \in N,(x) \chi=(s, y)$ with $s \in[0,1]$ and $y \in M$, and we set $(x)^{4} g^{\prime}=(s,(y)(i(s))) \chi^{-1}$. For $x \in{ }^{4} M \backslash N$ we set $(x)^{4} g^{\prime}=(x) \theta^{4} g \theta^{-1}$. Then ${ }^{4} g^{\prime}$ extends $g^{\prime}$ to ${ }^{4} M$.

\subsection{A special diffeomorphism $F$}

We are interested in the action on $\Psi$ of the diffeos $f_{e} \equiv g_{\gamma_{e}}$ and $f_{2 \pi} \equiv g_{\gamma_{2 \pi}^{1}}$ (see section 2) of $M$. We recall here the notion of the 'development' of a diffeo by a sequence of manifolds. For more details see [2,3]. $M$ is constructed by taking $\mathbb{R}^{3}$ and cutting out two polyhedra and making identifications. We construct a continuous sequence of such manifolds, cutting out the polyhedra in slightly different positions each time, the final one being $M$ again, the sequence thus being a 'loop of manifolds' $\dagger$. Each manifold in the sequence, $M(s)$, is diffeomorphic to $M$, so there exists a continuous sequence of diffeos $f(s): M \rightarrow M(s), s \in[0,1]$. The final diffeo $f(1)$ is a diffeo from $M$ to itself and we say that it is developed by the loop $M(s)$. Every diffeo is developed by some loop and two diffeos developed by the same loop are isotopic (i.e. there exists a continuous sequence of diffeos that interpolates between them) as are diffeos developed by homotopic loops of manifolds.

Suppose we choose, as a fiducial point, $\tilde{q}_{0}$, on the fibre, a metric which is flat outside two 2 -spheres, each surrounding one of the cut-out polyhedra, such that the isometry between the metrics inside the spheres is realized by translation through the flat region. Then the diffeo $f_{e}$ is developed by the sequence of manifolds which begins and ends with $M$ and in which the polyhedra are cut out at positions which move gradually around each other (with fixed orientation with respect to infinity) until they have swapped places. $f_{2 \pi}^{i}, i=1,2$, is developed by the sequence of manifolds, beginning and ending with $M$, in which one polyhedron is cut out at gradually rotated positions until it has rotated a whole turn and the

$\dagger$ That this sequence of manifolds is continuous in some appropriate sense seems clear, though this statement cannot as yet be given a precise meaning, since no topology on the space of manifolds has been exhibited with respect to which continuity could be defined. 


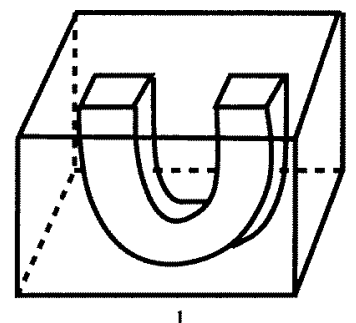

1

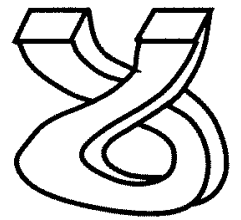

4

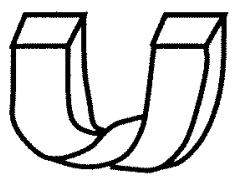

7
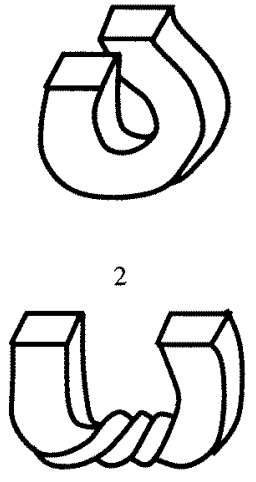

5

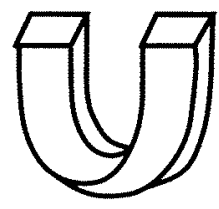

8
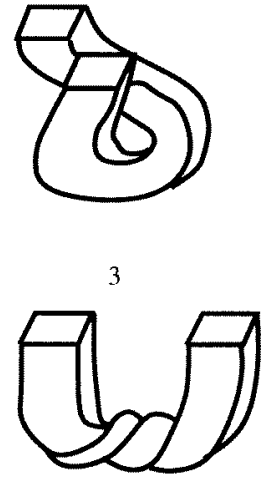

6

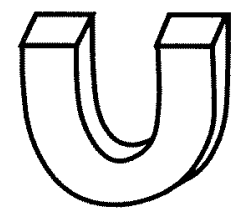

9

Figure 2. These are nine snapshots of the sequence of manifolds that develops the extension of $F$ to ${ }^{4} M$ (here represented as three dimensional). Only the cut-out tube is drawn in $2-9$, the surrounding manifold is implied.

other is cut out in the same fixed position all the time. It is clear that these diffeos represent physical exchange and $2 \pi$-rotation for the metric $\tilde{q}_{0}$. Let $F=f_{e} f_{2 \pi}^{1}$.

We saw that a diffeo acts trivially on $\Psi$ if it is extendible to ${ }^{4} M$. We now show that $F$ is extendible. To do so we exhibit the sequence of 4-manifolds which develops the extension. It starts with ${ }^{4} M$, and in the sequence the ends of the cut out tube swap positions, and then one end rotates around by $2 \pi$. The cut out tube itself gets twisted and then untwisted in the process so that the final manifold is ${ }^{4} M$ again. Figure 2 is a $2+1$ depiction of the sequence.

To see that it also works in $3+1$, let us first re-express the $2+1$ pictures in terms of 'framed curves'. We regard the pictures in figure 2 as manifolds induced from framed curves in the $t<0$ portion of $\mathbb{R}^{3}$. The curve itself gives the location of the tube, and the framing tell how it 'twists'. A framing just means attaching to each point of the curve a pair of labelled unit vectors orthogonal to the curve, and one can cut out the polyhedron and make identifications appropriate to a given prime at each point of the framed curve in a canonical manner.

Now, $F$ (still in $2+1$ dimensions) is developed by a sequence of manifolds, which under our correspondence, would be a loop in the space of pairs of 'framed points' (i.e. just frames) in $\mathbb{R}^{2}$. The framed points swap places without rotating and then one of them rotates by $2 \pi$. What figure 2 effectively shows is how to extend this sequence of frame pairs to a sequence of framed curves. But this gives us a sequence of 3-manifolds, whose boundaries develop $F$ (a diffeo of $M$ ); hence the diffeo of $M$ that they develop extends $F$ from $M$ to ${ }^{3} M$. 
So far we have just reformulated the $2+1$ proof. The generalization to any higher dimension is simple: just regard $\mathbb{R}^{3}$ as a subspace of $\mathbb{R}^{n-1}$ and complete the 2-frames to $(n-2)$-frames by adding a constant $(n-4)$-frame in the orthogonal directions. Thus the exchange-cum- $2 \pi$-rotation of the two frames in $\mathbb{R}^{n-1}$ extends to a continuous deformation of the framed U-curve in $\mathbb{R}^{n}$. As before we can glue a fixed (non-chiral) geon onto the framed curves to turn the loop of framed curves into a loop of manifolds which develops the extension of $F$ to ${ }^{n} M$.

We note that figure 2 is slightly misleading in that it actually matters in $2+1$ dimensions which way the end of the tube is rotated: one way the tube untwists, the other way the tube becomes more twisted. In $3+1$ this is not the case since a $4 \pi$-rotation is connected to the identity.

We have now shown that the diffeo $F$ acts trivially on any $\Psi$ of the form (3.1).

\subsection{The mapping class group}

Although it is clear that the diffeo $F$ is intimately connected with spin and statistics, we are still some way from a spin-statistics theorem. For one thing, the physical exchangecum- $2 \pi$-rotation loop $\Gamma=\gamma_{e} \gamma_{2 \pi}^{1}$ only acts on a wavefunction peaked around a two-geon configuration, $q$, as described in section 2.2. Even then, the loop $\Gamma$ only corresponds to the diffeo $F$ for a particular representative metric of $q$. To proceed, we will require more information about how other elements of the mapping class group, $G$, act on wavefunctions such as (3.1).

Relative to a presentation of $M$ as a connected sum, $G$ is generated by three sorts of elements (see, for example, [3]): (i) the generators of the internal diffeos of one of the primes; (ii) the exchange diffeo and (iii) the slide of one prime through the other. In fact, $G$ takes the form $G \simeq\left(S \ltimes G_{\text {int }}\right) \ltimes E$, where $S$ is the normal subgroup generated by the slide, $G_{\text {int }}$ is the internal group, $E \simeq Z_{2}$ is the subgroup generated by the exchange alone and $\ltimes$ denotes semidirect product [4]. Further, $G_{\text {int }}=G_{1} \times G_{2}$, where $G_{1}$ and $G_{2}$ are the internal groups of each separate geon and are isomorphic, which isomorphism is realized by translation, due to the particular presentation we have chosen.

A slide can be visualized by imagining one prime shrunk down to a tiny size and moved around some fixed non-contractible loop through the other one so that the resultant diffeo is the identity in the interior of some 2-sphere surrounding the geon doing the sliding. There is a twofold ambiguity in this definition of the slide which comes from the fact that the prime that is being slid can undergo a $2 \pi$-rotation while it is on its journey. (This ambiguity can be removed by specifying that the orientation of the slid prime be fixed with respect to some background field of frames.)

Suppose $[s]$ is the isotopy class of the slide diffeo. Consider $s \in[s]$ such that $s$ is the identity outside some embedded $S^{2}$ in $M$ surrounding the two primes. Extend the $S^{2}$ to an embedded cylinder $S^{2} \times[0,1]$ in ${ }^{4} M$ as shown in figure 3 and consider ${ }^{4} M^{\prime}$, the compact manifold with boundary, formed by cutting off ${ }^{4} M$ outside the cylinder. Let $M^{\prime}=\partial^{4} M^{\prime}$ so that $M^{\prime} \simeq P \# P$.

Then $s$ can be extended trivially to an element of $\mathrm{D}_{B}\left(M^{\prime}\right)$, the group of diffeos of $M^{\prime}$ which fix the bottom and sides of $M^{\prime}$. This extended $s$ can also be regarded as an element of $\mathrm{D}\left(M^{\prime}\right)$, the group of diffeos of $M^{\prime}$. We claim that, within $\mathrm{D}\left(M^{\prime}\right), s$ is in fact isotopic either to the identity or to the $2 \pi$-rotation of one of the primes (this being the twofold ambiguity in the definition of the slide mentioned above). The reason is that the 2-sphere separating the two primes in $M^{\prime}$ is unique (up to a homotopy). The slide is the 


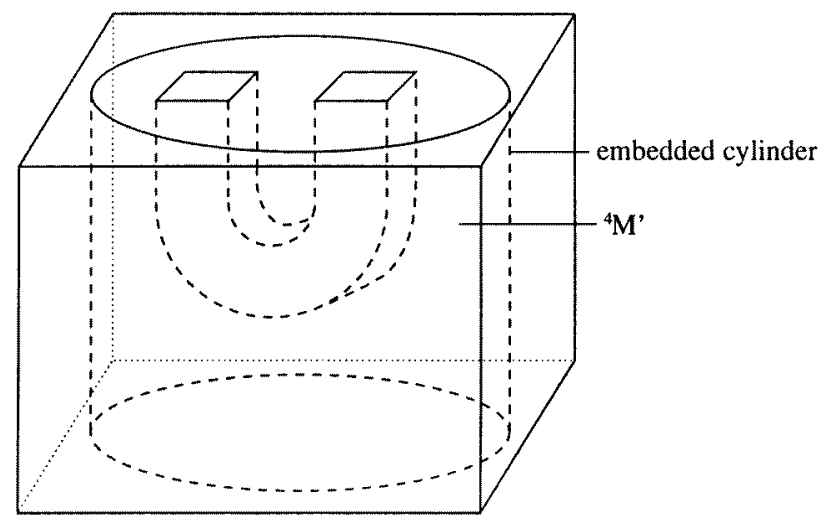

Figure 3. ${ }^{4} M^{\prime}$. Strictly speaking, for ${ }^{4} M^{\prime}$ to be a differentiable manifold, its 'edges' must be smoothed.

identity inside a 2-sphere surrounding one prime, which we can take to be the separating 2 -sphere. The slide must therefore be isotopic to an internal diffeo of the other prime. A slide, however, has a characteristic action on the generators of the fundamental group of $M^{\prime}$. In particular, it leaves the generators which thread the 'prime through which the other is slid' invariant. The only internal diffeo which does this is the $2 \pi$-rotation. Hence the slide is isotopic either to the identity or to the $2 \pi$-rotation in $\mathrm{D}\left(M^{\prime}\right)$. We assume that we have chosen the slide to be the one isotopic to the identity in $\mathrm{D}\left(M^{\prime}\right) \dagger$.

Now, any diffeo $g \in \mathrm{D}_{B}\left(M^{\prime}\right)$ which is isotopic to the identity in $\mathrm{D}\left(M^{\prime}\right)$ extends to a diffeo of ${ }^{4} M$. The extension is constructed by specifying it to be the identity on ${ }^{4} M \backslash N$ where $N$ is an open neighbourhood of $M^{\prime}$ in ${ }^{4} M^{\prime}$. Within the neighbourhood we use the isotopy sequence between $g$ and the identity in $\mathrm{D}\left(M^{\prime}\right)$ to construct the extension in the usual way. Thus we have that the slide $s$ extends to ${ }^{4} M$ and so $[s]$ acts trivially on $\Psi$. Similarly the entire normal subgroup $S$ generated by $[s]$ fixes $\Psi$ since any conjugate of something isotopic to the identity or product of things isotopic to the identity is also isotopic to the identity and therefore extends to ${ }^{4} M$.

So much for the slide. We can also derive relations between the action of the internal diffeos of one prime and the action of the internal diffeos of the other on $\Psi$. An internal diffeo of 'prime number one', followed by an internal diffeo of 'prime number two' which undoes the twisting of the U-tube caused by the first diffeo, will leave $\Psi$ invariant. Now with respect to our presentation, $G_{1}$ and $G_{2}$ are isomorphic via translation $a$ : $G_{1} \rightarrow G_{2}$, and (since $P$ is non-chiral) we can arrange that there exists a second, 'mirror' isomorphism, $b: G_{1} \rightarrow G_{2}$ which is given by reflection in the plane of symmetry between the two cut-out polyhedra of $M$. Then the element $\left(g_{1}, g_{2}\right) \in G_{1} \times G_{2}$ extends if $g_{2}=b\left(g_{1}\right)$, and so $\Psi$ is fixed by $\left(g_{1}, b\left(g_{1}\right)\right)$.

$\dagger$ We thank Bob Gompf of the University of Texas at Austin for pointing out the triviality of the slide to us and providing this argument. Strictly speaking, we should also check that the $2 \pi$ rotation $R$ of a single prime does not belong to the slide subgroup $S$, when the rotation ambiguity in the slide is resolved as above. But slide trivial in $\mathrm{D}\left(M^{\prime}\right) \Rightarrow S$ trivial in $\mathrm{D}\left(M^{\prime}\right)$, whereas $R$ is not trivial in $\mathrm{D}\left(M^{\prime}\right)$ (for spinorial $P$ ); hence $R \notin S$. Finally, we note that the triviality of the slide in $\mathrm{D}\left(M^{\prime}\right)$ can also be established directly by constructing an explicit deformation of it to the identity. 


\section{The spin-statistics theorem}

Consider the space $\Lambda$ of all wavefunctions on the covering space $\tilde{Q}$. Given a state vector in $\Lambda$, we can imagine decomposing it into a superposition of components, each of which lives in a primary subspace $\Lambda_{\rho}$ of $\Lambda$, where a primary subspace is the direct sum of a number of copies of a single unitary irreducible representation (UIR) $\rho$ of $G$. The projection of a wavefunction onto the primary subspace corresponding to a particular UIR $\rho$ is achieved using the (un-normalized) operator:

$$
P_{\rho}=\sum_{g \in G} \chi_{\rho}\left(g^{-1}\right) g
$$

where $\chi_{\rho}$ is the character of the representation $\rho$. Note that $P_{\rho}$ commutes with every element of $G$.

We have seen that the normal subgroup $S$ of slides leaves our state $\Psi$ invariant. Hence the only primary components that can occur in $\Psi$ are those corresponding to UIRs in which the slides are represented trivially.

Proof. Let $\Pi$ project $\Lambda$ onto any irreducible component $\Lambda_{\rho 0} \subseteq \Lambda$. Since $\Pi \llbracket G$, we can write its action consistently on the left. Also let $\Psi_{0}=\Pi \Psi$. Then $\forall g \in G, \forall s \in S$ we have

$$
\Psi_{0} g s=\Psi_{0}\left(g s g^{-1}\right) g \equiv \Psi_{0} s^{\prime} g
$$

where s' $\in S$ since $S$ is normal; and further

$$
\Psi_{0} s^{\prime}=(\Pi \Psi) s^{\prime}=\Pi\left(\Psi s^{\prime}\right)=\Pi \Psi=\Psi_{0} ;
$$

hence $\left(\Psi_{0} g\right) s=\Psi_{0} g$. But the $\Psi_{0} g$ span the irreducible subspace $\Lambda_{\rho 0}$, whence $s$ must act as the identity on $\Lambda_{\rho 0}$. Finally, since the choice of $\Lambda_{\rho 0} \subseteq \Lambda_{\rho}$ was arbitrary, it follows that $s$ must act trivially on $\Lambda_{\rho}$ itself.

The finite-dimensional UIRs in which the slides are represented trivially have been classified in [4]. They are specified by: (i) a choice of an (unordered) pair $\left(\rho_{1}, \rho_{2}\right)$ of finite dimensional UIRs of the internal group of a single prime (say $G_{1}$ ) and (ii) a choice of sign for the exchange. At the end of the previous section we showed that certain elements $\left(g_{1}, g_{2}\right) \in G_{1} \times G_{2}$ act trivially on $\Psi$. From this we can also deduce a condition on $\rho_{1}$ and $\rho_{2}$, namely that they are 'CP conjugate' representations, these being defined by $\rho_{1}(g)=\rho_{2}\left(a^{-1} b(g)\right)$. If $\rho_{1}$ and $\rho_{2}$ are inequivalent UIRs then the quantum geons they describe will be distinguishable particles, and no question of statistics will arise. If, on the other hand, $\rho_{1}$ and $\rho_{2}$ are equivalent, then the geons will be identical, so let us now concentrate on those particular primary subspaces.

Let us further assume that the representation $\rho_{1}$ that determines the physical type of the geons is Abelian, in which case $\rho$ itself is also Abelian [4]. This means that $\rho$ represents every element of the mapping class group $G$ by a pure number. Now consider the 'component' $\Psi_{\rho}:=\Psi P_{\rho}$ of $\Psi$ in the subspace $\Lambda_{\rho}$. We have, $\Psi_{\rho} F=\Psi P_{\rho} F=$ $\Psi F P_{\rho}=\Psi P_{\rho}=\Psi_{\rho}$. Unless $\Psi_{\rho}$ vanishes, this means that $F$ acts in $\Lambda_{\rho}$ as the number +1 . In particular, this implies that if $\Phi \in \Lambda_{\rho}$ is any wavefunction in the subspace on which the loop $\Gamma$ can act (namely a wavefunction peaked on a geometry describing two identically configured well separated geons), then $\Gamma$ acts trivially, or equivalently the exchange and rotation loops act identically to each other.

Therefore, if a quantum sector carries Abelian internal representations $\rho_{1}=\rho_{2}$, and if $\Psi$ has support on that sector, then that sector respects the spin-statistics correlation in the following sense. For any state $\Psi$ in the sector on which the exchange and $2 \pi$-rotation 
loops act, both loops act identically. We will call such sectors spin-statistics respecting (and we note that all other sectors which carry an Abelian representation of the MCG are spin-statistics violating).

Although this proof involves some subtle points, its main idea is simply expressed. Our choice of a particular presentation of the manifold $M$ has the effect of labelling the geons, and wavefunctions $\Psi: \tilde{Q} \rightarrow \mathbb{C}$ can therefore be thought of as functions on a configuration space of labelled particles. Then (under appropriate conditions, these providing the subtleties), the diffeomorphism $f_{e}$ just represents exchange of labels, while $f_{2 \pi}^{i}$ represents $2 \pi$ rotation of the geon labelled $i$. The relation $\Psi F=\Psi$ then says that exchange of labels is equivalent to rotation of the first geon, which is the spin statistics correlation.

We are apparently unable to say anything about the quantum sectors corresponding to non-Abelian internal representations. This might have been expected, since geons carrying non-Abelian representations of their internal diffeomorphism groups possess nongeometrical internal states (the phenomenon of 'quantum multiplicity'), and consequently, even when they respect the spin-statistics correlation, one can construct states on which the exchange loop $\Gamma$ acts as minus one. For example, suppose the geons are bosons. Take the state in which geon A is in internal state 'up' and geon B is in internal state 'down' and superpose this with the state in which A is down and B is up, with a relative minus sign. Then the exchange will take this state to minus itself, but the geons are the epitome of boson-hood nevertheless. Excluding a non-Abelian spin-statistics violating sector will thus require a stronger condition than just the equality $\Psi F=\Psi$. (In fact, even the question of which of the words 'boson' and 'fermion' to attach to which sectors can become confusing in some non-Abelian cases.)

There are primes for which our result is a more complete spin-statistics theorem than for others, because their internal diffeomorphism groups are already Abelian, and therefore the restriction to Abelian representations is no restriction at all. These are the lens spaces $L(p, q)$ with $q^{2}=-1 \bmod p$ (the restriction on $p$ and $q$ is necessary and sufficient for the lens space to be non-chiral). Their internal group is $Z_{2}$, the non-trivial element being a $\pi$ rotation, so they are tensorial. (A result due to Witt [9] states that they are the only non-chiral primes (except for the handle) with a finite internal group. As far as we know, it is an open question as to whether there exists a non-chiral prime whose internal group is infinite Abelian.) So lens space geons, pair-created via the cobordism ${ }^{4} M$, must be bosons.

In using the operator (4.1) to decompose our U-tube engendered wavefunction $\Psi$ into primary components, we have been rather cavalier about the fact that the group $G$ is infinite and discrete. This causes two main problems. First, there is no reason for $P_{\rho} \Psi$ to be normalizable, and it certainly cannot be normalizable in the most important cases, where $\rho$ is finite dimensional. Second, infinite discrete groups commonly possess primary representations of types II and III, and when this is the case for $G$, the operator $P_{\rho}$ belonging to such primaries does not seem to be well defined, even formally (especially for type III). Indeed, the decomposition into irreducibles of type II and III representations is not unique, and a type II or III primary can apparently not be associated naturally with any UIR at all.

These problems arise partly because we have chosen to use covering space quantization as a familiar setting in which to discuss spin and statistics. In a frozen topology setting, one could solve the normalization problem by treating each UIR as an inequivalent quantum theory (described in terms of a vector bundle) and normalizing state vectors separately within each sector. The type II and III representations could be avoided by restricting 
only to finite-dimensional UIRs. Neither device is possible here, since we have topology change. However, we believe that our work can (and should) be expressed solely in terms of spacetime histories. In a $\mathrm{SOH}$ formulation, normalization and restriction to finite dimensional UIRs appear to present no special difficulties. We discuss the SOH further in the next section.

\section{Conclusions}

In summary, a wavefunction $\Psi$ which is given by a functional integral over geometries on a 'U-tube pair-creation cobordism' ${ }^{4} M$ has no support on certain 'theta sectors' of canonical quantum gravity, namely those corresponding to spin-statistics violating Abelian representations of the MCG. In particular, lens space geons $L(p, q)$ with $q^{2}=-1 \bmod p$, pair created via the cobordism ${ }^{4} M$, satisfy a spin-statistics correlation. The lens spaces are tensorial-the $2 \pi$-rotation of a lens space is trivial-so the result rules out the possibility that they are fermions.

We had restricted ourselves to orientable, non-handle geons but we can generalize our calculation to include non-orientable non-handles. In this case, the condition of non-chirality is not meaningful and the U-tube is always a cobordism between $\mathbb{R}^{3}$ and $\mathbb{R}^{3} \# P \# P$ when $P$ is non-orientable. The steps of our calculation follow just as for the orientable case.

How do we re-express our work in spacetime terms? A sketch of the fixed-topology case was given in [4]. In the $\mathrm{SOH}$ framework the fundamental dynamical input is a rule attaching a quantum amplitude to each pair of truncated histories which 'come together' at some 'time' $[5,10,11]$. Let us call such a pair a 'Schwinger history' for short, and its underlying manifold a 'Schwinger manifold'. In the case of quantum gravity, a truncated history is a Lorentzian manifold with final boundaryt (and possibly initial boundary depending on the physical context), and the 'coming together' means the identification or 'sewing together' of the final boundaries. Now different ways of sewing are possible, related to each other by large diffeomorphisms of the final boundary. In general such a re-identification may or may not lead to a diffeomorphic Schwinger manifold, but it never will if we restrict ourselves to product spacetimes of the form $\mathbb{R} \times{ }^{4} M$, i.e. if we exclude topology change (and if we limit ourselves to diffeomorphisms vanishing on any initial boundaries which may be present). In this case, the mapping class group $G$ of ${ }^{4} M$ acts freely and transitively (albeit non-canonically) on the set of Schwinger manifolds.

Now, without disturbing the classical limit of the theory or the local physics, we can multiply the amplitude of each Schwinger history by a complex 'weight' $w$ depending only on the topology of the underlying manifold (and on the two initial metrics, if initial boundaries are present). Somewhat analogously to [12], one can then argue that consistency requires that these complex weights transform under some unitary representation of $G$, and that sets of weights belonging to disjoint representations 'do not mix'. The pure cases are then the UIRs, and we arrive again at the conclusion that each distinct UIR of the mapping class group yields an inequivalent version or 'sector' of quantum gravity with frozen topology. Notice here that the weight function $w: G \rightarrow \mathbb{C}$ need not (and in general will not) be square summable over $G$ (the trivial UIR of $G$ corresponds to $w(g) \equiv 1$ for example). Thus there is no apparent normalization problem in the $\mathrm{SOH}$ formulation.

Now let us bring in topology change and consider a Schwinger pair of U-tube cobordisms. Our spin-statistics result translates into the statement that it would be

$\dagger$ This final boundary corresponds to the spacelike slice ${ }^{4} M$ of the canonical formulation. 
inconsistent to try to use for the Schwinger manifolds one obtains from the different attaching maps, a set of weights carrying an Abelian spin-statistics violating UIR of $G$. (We would find that we were trying to attach different weights to manifolds in the same diffeomorphism equivalence class.) Such spin-statistics violating possibilities are thus ruled out when one allows topology change.

One might contemplate enhancing the status of our result by strengthening the restriction on UIRs of $G$ from finite dimensionality down to one dimensionality (i.e. by admitting only Abelian UIRs). This might be going too far, however, not only because there is no evident physical basis for such a drastic restriction, but also because every prime 3manifold whose internal group is known to us lacks Abelian spinorial representations. A restriction to Abelian representations, therefore, might rule out spinorial geons altogether, which would not be desirable. However, in some sense of the word 'most', the internal group remains unknown for most prime 3-manifolds-the mysterious and multitudinous hyperbolic primes-and they might include among them primes with Abelian spinorial representations. We do not know how likely this is, but physically it would seem hard to explain the appearance of spin $\frac{1}{2}$ without being able to trace it to some underlying 'hidden' degrees of freedom which, in turn, would be reflected in the quantum multiplicity associated with non-Abelian representations. In this sense we can use physical reasoning to 'predict' something about 3-manifold topology: there should be no prime whose MCG admits one-dimensional spinorial UIRs.

We see this work as an indication that there is a spin-statistics theorem 'trying to get out' of a sum-over-histories formulation of quantum gravity, and that it seems indeed to be intimately connected with the process of pair-creation, as predicted. We do not think, however, that a full spin-statistics theorem (including results for chiral geons and 'primordial' geons) can be proved without extra input to the SOH rules, such as that suggested in [3].

\section{Acknowledgments}

It is a pleasure to thank Lee Brekke, Andrew Chamblin, John Friedman, Nico Giulini, Bob Gompf, Jorma Louko, Trevor Samols and Don Witt for help and interesting discussions. RDS was supported in part by NSF grant no PHY-9307570 and HFD by the DOE and NASA grant NAGW-2381 at Fermilab, by NSF grant no PHY-9008502 at Santa Barbara and by the US Department of Energy under grant no DE-FG03-92-ER40701 at Caltech. She also thanks the Instituto de Ciencias Nucleares at the Universidad Nacional Autonoma de Mexico for hospitality during the completion of this work.

\section{References}

[1] Friedman J L and Sorkin R D 1982 Gen. Rel. Grav. 14615

[2] Sorkin R D 1986 Introduction to topological geons Topological Properties and Global Structure of SpaceTime, Proc. NATO Advanced Study Institute on Topological Properties and Global Structure of Space-Time (Erice, 1985) ed P G Bergmann and V De Sabbata (New York: Plenum)

[3] Sorkin R D 1989 Classical topology and quantum phases: quantum geons Geometrical and Algebraic Aspects of Nonlinear Field Theory, Proc. Meeting on Geometrical and Algebraic Aspects of Nonlinear Field Theory (Amalfi, 1988) ed S De Filippo, M Marinaro, G Marmo and G Vilasi (Amsterdam: North-Holland)

[4] Sorkin R D and Surya S An analysis of the representations of the mapping class group of a multi-geon three-manifold Preprint gr-qc/9605050

[5] Sorkin R D 1994 On the role of time in the sum-over-histories framework for gravity Int. J. Theor. Phys. 33 523-34 (presented to the Conf. on The History of Modern Gauge Theories (Logan, UT) 1987) 
[6] Mackey G W 1976 Theory of Unitary Group Representations (Chicago, IL: University of Chicago Press)

[7] Sorkin R D 1988 Commun. Math. Phys. 115421

[8] Hartle J B and Witt D M 1988 Phys. Rev. D 372833

[9] Witt D Private communication

[10] Daughton A, Louko J and Sorkin R D Initial conditions and unitarity in unimodular quantum cosmology Proc. 5th Canadian Conf. on General Relativity and Relativistic Astrophysics (Waterloo, 1993) (Singapore: World Scientific) Preprint gr-qc/9305016

[11] Sorkin R D Quantum measure theory and its interpretation Proc. 4th Drexel Symp. on Quantum Nonintegrability: Quantum Classical Correspondence (Philadelphia, PA, 1994) ed D H Feng and B-L Hu (International Press) pp 205-227 Preprint gr-qc/9507057

[12] Laidlaw M G C and DeWitt C M 1971 Feynman functional integrals for systems of indistinguishable particles Phys. Rev. D 3 1375-8 